\title{
Whipple's Disease
}

National Institute of Neurological Disorders and Stroke (NINDS)

\section{Source}

National Institute of Neurological Disorders and Stroke (NINDS). Whipple's Disease

Information Page.

Whipple's disease is a multi-system infectious bacterial disease that interferes with the body's ability to metabolize fats. Caused by the bacterium Tropheryma whipplei, the disorder can affect any system in the body, including the brain, eyes, heart, joints, and lungs, but usually occurs in the gastrointestinal system. Neurological symptoms occur in up to 40 percent of individuals and may include dementia, abnormalities of eye and facial muscle movements, headaches, seizures, loss of muscle control, memory loss, weakness, and vision problems. Gastrointestinal symptoms may include diarrhea, weight loss, fatigue, weakness, and abdominal bleeding and pain. Fever, cough, anemia, heart and lung damage, darkening of the skin, and joint soreness may also be present. The disease is more common in men and neurological symptoms are more common in individuals who have severe abdominal disease. Rarely, neurological symptoms may appear without gastrointestinal symptoms and can mimic symptoms of almost any neurologic disease. 